\section{Prevalencia de parasitismo intestinal en niños quechuas de zonas rurales montañosas de Ecuador}

Diversos estudios epidemiológicos abordan el tema de la prevalencia del parasitismo intestinal en Ecuador y otros países de América del Sur, pero pocos han estudiado el parasitismo en niños preescolares quechuas.

En este trabajo se determinó la prevalencia de varias infecciones parasitarias en niños pequeños de comunidades rurales quechuas que viven en las montañas de la provincia de Chimborazo, en la región central de Ecuador. Además, se analizó la relación entre algunas variables sanitarias (el uso de letrinas, la disponibilidad de métodos adecuados de almacenamiento y tratamiento de agua y la aplicación de proyectos comunitarios para proteger las fuentes de agua potable) y las enfermedades y la prevalencia de parasitismo infantil. Se buscó una gran variedad de parásitos, la mayoría de ellos patógenos, que se transmiten por diversas vías, como el agua, los alimentos, el suelo y las heces fecales. Se analizó el nivel de acceso de estas comunidades al agua potable, al agua necesaria para su higiene y a sistemas sanitarios de eliminación de los desechos fecales, ya que todos estos factores pueden incidir en el nivel de infestación.

Se analizaron muestras de heces fecales de $112(75,2 \%)$ de los 149 niños residentes en las comunidades con proyectos de higienización y protección del agua potable y de $91(63,2 \%)$ de los 144 niños de las comunidades sin ese tipo de proyectos. En general, 85,7\% de las muestras presentaban al menos uno de los 10 parásitos estudiados y 63,4\% contenían dos o más especies de parásitos. La prevalencia general fue de $57,1 \%$ de Entomoeba histolytica o Entomoeba dispar, 35,5\% de Ascaris lumbricoides, 34,0\% de Escherichia coli, 21,1\% de Giardia intestinalis (lamblia), 11,3\% de Hymenolepis nana, 8,9\% de Cryptosporidium paroum, 1,7\% de Chilomastix mesnili, 1,0\% de Hymenolepis diminuta, 0,7\% de Strongyloides stercoralis y $0,5 \%$ de Trichuris trichiura. Se encontraron protozoos en $78,3 \%$ de las muestras $y$ helmintos en $42,4 \%$. No se encontraron diferencias significativas entre el número de casos informados de enfermedades (diarrea, fiebre, infecciones respiratorias, vómitos y otras) según los parásitos detectados, excepto un ligero incremento en el número informado de diarreas en el mes ante- rior en los niños con infección por E. histolytica o E. dispar $(65,2 \%$ contra $49,6 \%$; $P=0,032)$.

La prevalencia de parásitos intestinales aumentó con la edad. En comparación con los otros niños, los de mayor edad (48-60 meses) presentaron una mayor probabilidad de tener al menos uno de los 10 parásito estudiados $(P<0,001)$ o más de uno de ellos $(P=0,002)$ que el resto. Esta tendencia fue significativa en los casos de E. histolytica o E. dispar $(P<0,001)$ y E. coli $(P=0,030)$. La tendencia fue de menor significación para $H$. nana $(P=0,068)$ y $G$. intestinalis $(P=0,070)$ y no fue significativa para $A$. lumbricoides $(P=0,386)$ y $C$. paroum $(P=0,407)$. Los niños de menor edad (12-23 meses) tuvieron una probabilidad significativamente mayor que los otros grupos de presentar diarreas en la semana y el mes previos ( $P=0,013$ y $P<0,001$, respectivamente).

No se encontraron diferencias significativas en la prevalencia de parásitos intestinales o diarrea y la participación en proyectos comunitarios de agua potable. El almacenamiento y el tratamiento del agua, el uso habitual de letrinas y la participación en proyectos comunitarios de agua potable no mostraron una asociación fuerte con la prevalencia de parásitos intestinales. El suelo sucio fue un factor de riesgo de la infección con E. histolytica o $E$. dispar y G. intestinalis.

Estos resultados indican que la instalación de fuentes limpias de agua potable y la disponibilidad de instalaciones sanitarias no garantizan por sí solas cambios significativos en la conducta higiénica y las prácticas sanitarias de la comunidad, por lo que es importante complementar estas intervenciones con un componente educativo-sanitario si se desea reducir la diarrea y el parasitismo intestinal en estas comunidades. (Jacobsen KH, Ribeiro PS, Quist BK, Rydbeck BV. Prevalence of intestinal parasites in young Quichua children in the highlands of rural Ecuador. J Health Pop Nutr. 2007;25(4):399-405.)

\section{Costos de la detección, el diagnóstico y la clasificación del cáncer de próstata en países industrializados: una referencia necesaria}

Debido a que en ningún país se cuenta con los recursos suficientes para cubrir todas las necesidades sanitarias, se hace imprescindible comprender los elementos que inciden en las estimaciones del costo de las enfermedades, de modo que se pueda 
disponer de la información adecuada para decidir si se debe invertir o no, por ejemplo, en sistemas y programas de detección precoz de esas enfermedades. Esto es particularmente importante en el caso del cáncer de próstata, no solamente por ser el segundo tipo de cáncer más frecuente en hombres, sino por los beneficios que pueden ofrecer su detección precoz y su oportuno tratamiento. No obstante, la efectividad de la detección temprana en la reducción de la mortalidad asociada con el cáncer de próstata es aún tema de debate.

Existen diversas estimaciones de los costos asociados con la detección, el diagnóstico y la clasificación del cáncer de próstata. Sin embargo, aún se desconocen los costos en recursos -el tiempo de trabajo del médico, los análisis de laboratorio, el tiempo que el paciente debe ausentarse del trabajo, entre otros - a tomar en cuenta para estas estimaciones.

En este trabajo se analizaron los costos de los recursos empleados en la detección, el diagnóstico y la clasificación por etapas del cáncer de próstata en los Estados Unidos de América y en qué medida esos costos difieren de los publicados en otros países industrializados.

Para ello se buscaron los artículos e informes sobre el cáncer de próstata publicados entre enero de 1980 y diciembre de 2003 e indizados en las bases de datos electrónicas MEDLINE, EMBASE y CINAHL. Se seleccionaron los artículos publicados en inglés, disponibles en texto completo, que contuvieran los datos sobre los costos de los recursos o los datos necesarios para estimar el costo de la detección, el diagnóstico y la clasificación de las etapas de cáncer de próstata. Se seleccionaron solamente los estudios realizados en países con economía de mercado consolidada. Para agrupar y analizar los datos obtenidos se utilizaron procedimientos estadísticos descriptivos, valores medios ponderados y el método de simulación Monte Carlo.

De los 262 estudios analizados, 28 cumplían con los criterios de selección (15 eran de los Estados Unidos y 13 de otros países industrializados). En los estudios realizados en los Estados Unidos se observó que el costo inicial combinado de los recursos para la detección mediante el antígeno prostático específico fue de US\$37,23 y para la detección mediante el examen rectal digital, de US\$31,77. En los estudios realizados en otros países industrializados, el costo inicial combinado de los recursos para la detección mediante el antígeno prostático específico fue de US\$ 30,92 y de US\$33,54 para la detección mediante el examen rectal digital. Se observó una gran variación en los costos de los recursos vinculados a los procedimientos de diagnóstico y de clasificación por etapas, tanto dentro de los Estados Unidos como en los otros países industrializados analizados.
Este estudio puede ser de gran utilidad para las autoridades sanitarias de otros países de la Región de las Américas, ya que por primera vez se ofrece un valor de base que puede emplearse como referencia al trazar políticas sanitarias relacionadas con el tamizaje - masivo o selectivo- de cáncer de próstata, siempre que se adapte a las condiciones locales. (Ekwueme DU, Stroud LA, Chen Y. Cost analysis of screening for, diagnosing, and staging prostate cancer based on a systematic review of published studies. Prev Chronic Dis. 2007;4(4). Hallado en http://www.cdc.gov/pcd/issues/2007/ oct/06_0051.htm. Acceso el 24 de enero de 2008.)

\section{Influencia de dos métodos de cálculo de las inequidades en salud: estudio en 22 países}

El efecto negativo de las inequidades socioeconómicas sobre la salud continúa siendo un tema prioritario en las agendas de las autoridades sanitarias de la mayoría de los países. Para los gobiernos y la comunidad internacional es importante y deseable poder medir y comparar la magnitud de las inequidades en salud - ya sea entre diferentes países o entre diferentes zonas o grupos de un mismo país-, así como evaluar la magnitud de los cambios ocurridos en esas inequidades en un período de tiempo dado.

Aunque existen muchas formas de evaluar el nivel de las inequidades en salud, las mediciones más empleadas son la razón de las tasas y la diferencia de las tasas. Estas mediciones se basan en la comparación (ya sea mediante la razón o la diferencia) de las tasas de mortalidad en relación con la posición socioeconómica. Para ello se comparan los valores de mortalidad correspondientes al segmento de mejor situación socioeconómica (por lo general el $20 \%$ superior) con la mortalidad correspondiente al segmento de peor situación socioeconómica (por lo general el 20\% inferior). A pesar de que estas mediciones no toman en cuenta toda la información disponible, son las más frecuentemente utilizadas por su sencillez y fácil uso.

En este trabajo se empleó información pública para analizar los niveles y las tendencias de la inequidad en salud a partir de los datos de mortalidad en menores de 5 años de 22 países clasificados como de ingresos bajos y mediano-bajos que aplicaron dos encuestas de población y salud entre 1991 y 2001. Se analizaron once países de África, seis de Asia y cinco de América Latina (Colombia, Guatemala, Haití, Nicaragua y Perú), que en su conjunto representan $27 \%$ de la población mundial.

Se observó que en ocasiones, la posición relativa de los países según su nivel de inequidad en un momento determinado varió en dependencia de la 